Anders Herlitz ${ }^{1}$

\title{
Nondeterminacy and Population Ethics
}

This paper synthesizes a general view out of Derek Parfit's last views on how to avoid the Repugnant Conclusion and presents the general features of a plausible theory of population ethics based on Parfit's suggestions. The paper argues that a plausible population axiology provides only partial orderings and implies that some outcomes are nondeterminate in their ranking. The paper shows, first, how the combination of what Parfit calls "imprecise equality" and the "Wide Dual Person-Affecting Principle" allows one to avoid both the Continuum Argument and the Improved Mere Addition Paradox. Second, the paper shows how this is enough to in principle also refute Gustaf Arrhenius's impossibility theorems. Third, the paper suggests that a plausible population axiology must allow for nondeterminacy, that whatever the substance of the axiology is, it can only provide partial orderings of outcomes, and that if we revise Arrhenius's adequacy conditions these can condition what a satisfactory population axiology looks like. Finally, the paper illustrates how one can apply normative theories that allow for nondeterminacy and also infer formal constraints on the theories in light of the consequences of their application.

\footnotetext{
${ }^{1}$ Institute for Futures Studies, anders.herlitz@iffs.se. Financial support by Riksbankens Jubileumsfond (the Swedish Foundation for Humanities and Social Sciences) is gratefully acknowledged.
} 
Derek Parfit has done more than anyone to identify notorious problems in population ethics. One of the most pressing issues that he has drawn our attention to is the difficulty of avoiding the so-called Repugnant Conclusion (Parfit 1982, 1984):

The Repugnant Conclusion: Compared with the existence of many people whose quality of life would be very high, there is some much larger number of people whose existence would be better, even though these people's lives would be barely worth living (Parfit 2016: 110, 2017: 153).

To grasp just how counterintuitive this position is it might help with some illustration. The Repugnant Conclusion, for instance, implies that compared with a population in which ten billion people flourish and lead lives filled with happiness, love, friendship and successful pursuit of worthwhile goals such as artistic creation, there is a much larger population in which all individuals lead lives which are barely worth living which is better. How could it be that a population - no matter its size in which each individual has no other pleasure than "muzak and potatoes" (Parfit 1984) or the equivalent of the benefits accrued to a "lizard basking in the sun" (Parfit 2016) is better than a very large population in which everyone flourishes? To take this position seems absurd, and to suggest that a world filled only with people with damp lives is better than a world filled only with people with happy lives seems repugnant. The fact that the former population - with people leading damp lives might be much, much bigger does not seem to affect our considered judgment that the latter population - with billions of people in happiness - is better.

Although the conclusion that the population with many damp lives is better to many indeed appears repugnant, several very compelling arguments support it. In particular, Parfit - and many with him - is troubled by two arguments that seem to give the view expressed in the Repugnant Conclusion very firm support: the Continuum Argument and different versions of the Mere Addition Paradox.

The Continuum Argument aims to establish that one must accept the Repugnant Conclusion if one makes pairwise comparisons of different populations the only difference between which is that one is significantly smaller but the individuals in it have slightly better lives. The argument relies on the following main premise:

$\varphi$ : Compared with the existence of many people who would all have lives that were equally worth living, there are some much larger numbers of people whose existence would be better, though these people would all have lives that would be slightly less worth living (Parfit 2016: 116). 
This premise has significant intuitive support. To see this, compare the following populations. Population A consists of ten billion people who all have lives that are perfectly healthy and filled with happiness and meaningful activities. Population $B$ consists of one hundred billion people who would all have lives that are filled with happiness and meaningful activities, but which are only almost perfectly healthy; the individuals in Population B all experience getting the flu one of the many winters they experience, and they all go through the quite unpleasant experiences associated with that. Intuitively, the fact that all the people in Population B need to endure a strike of the flu does not mean that Population $\mathrm{A}$ is better than Population B. Population B is ten times as big and the wellbeing of the people in Population B is nearly as good as the wellbeing of the people in Population A. Similar thought experiments seem possible for all population sizes and all levels of wellbeing, and thus the general claim expressed in $\varphi$ seems very plausible.

If $\varphi$ is true and if the better than relation is transitive (something some prominent philosophers have disputed, but which I will assume in this paper, cf. Rachels 1998; Temkin 1987, 2012), one must accept the Repugnant Conclusion. To see this, consider a continuum of populations, A-Z, in which the first population, A, consists of many people with very good lives, the second population, $\mathrm{B}$, is much larger but the people in it have slightly worse lives, Population $\mathrm{C}$ consists of much more people than Population B, but the people in it have slightly worse lives, and so on until we reach Population $\mathrm{Z}$ which is an astronomically large population with lives that are barely worth living. Since $\varphi$ tells us that an immediately successive population in this continuum is better than its predecessor, and since better than is a transitive relation, we must conclude that $\mathrm{Z}$ is better than $\mathrm{A}$. Consider a simple illustration in which the width of the blocks shows the size of the populations and the height shows the quality of people's lives:
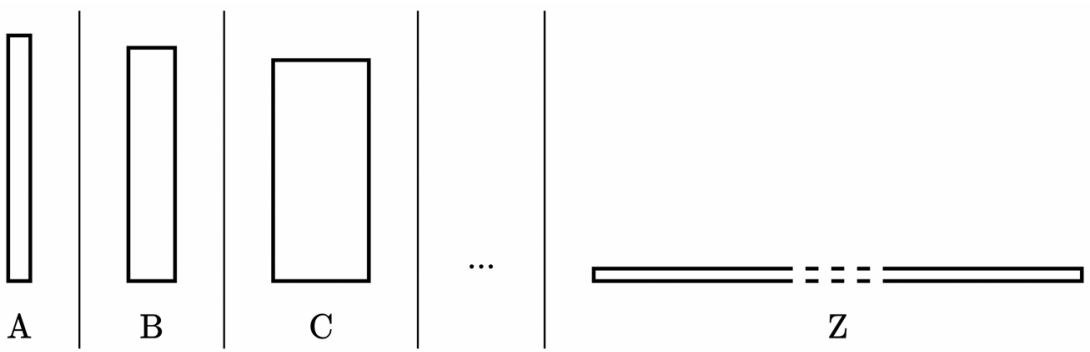

Figure 1 
By only assuming that there is some population (however big) with individuals that lead lives that are only slightly worse that is better than a population with many people with good lives and that the better than relation is transitive, we are forced to conclude that A is worse than Z. That is the Repugnant Conclusion.

In his early writings on this topic, Parfit also expressed worries that the Repugnant Conclusion could not be avoided because of what he called the Mere Addition Paradox (Parfit 1982, 1984; Temkin 1987). I will here focus on a particular version of the Mere Addition Paradox. I take this version to be a stronger version of the initial argument, and it is the version Parfit discusses in his later writings (Parfit 2016). I will call this the Improved Mere Addition Argument. This argument resembles the Continuum Argument in that it makes use of the transitivity of the better than relation and comparisons of populations that are relatively close to each other in a continuum like the one described above. Contrary to the Continuum Argument, however, it does not rely merely on intuitive support for premise $\varphi$. Instead, it provides reasons to accept $\varphi$ that are supported by other intuitions, expressed in premises $\Pi$ and $\omega$ :

п: Compared with the existence of many people who would all have lives that were equally worth living, there is some larger population that consists of the very same people with even better lives as well as some additional people with lives that are slightly worse than the lives of the people in the first population that would be better.

Contrary to the main premise of the Continuum Argument, this premise does not appeal to intuitions about trade-offs between the number of people living and the quality of their lives. Instead, $\Pi$ only states that it would be better if the lives of everyone would be better even if this means that the population grows so that there is an additional number of people with lives that are slightly worse than the first group. This seems indisputable. Consider, for instance, Populations A and B above, where the only difference is that the people in Population B contracts the flu at some point in their lives. It seems ludicrous to deny that if one could improve the lives of all the people in Population A so that they would be even better by adding all the people in Population B and thereby create Population A+ then Population A+ would be better than Population A. Similar thought experiments seem possible for all population sizes and all levels of wellbeing, and thus the general claim expressed in $\Pi$ seems very reasonable. 
$\omega$ : Compared with the existence of many people with very good lives and many people with slightly worse lives, there is some population that consists of the same people where all lives are equally good, and where the total amount of wellbeing is greater that would be better.

This premise appeals to both inequality aversion and the preference for increasing the total amount of wellbeing. The underlying idea is that a larger amount of wellbeing that is more equally distributed is preferable to a smaller amount of wellbeing less equally distributed. Since the inequality aversion here is expressed in a very weak form (more equal distributions are favored when this increases total amount of wellbeing), this premise should be appealing to those who value equality, to those who value wellbeing increases and to those who value both equality and wellbeing increases. $\omega$ also appears intuitively plausible. Consider Population A+ again (the improved Population A together with Population B). Compared to A+, a population that consists of one group of people who lead phenomenally good lives and who never contracts the flu and a different group of people who lead phenomenally good lives but who contracts the flue and thus are sick for a couple of days, an alternative population that consists of both of these groups of people but where no one contracts the flu but everyone gets a minor stomach upset for a couple of hours at one point in their lives is better. Again, similar thought experiments seem possible for all population sizes and all levels of wellbeing, and thus the general claim expressed in $\omega$ seems very reasonable.

Accepting $\Pi$ and $\omega$ amounts to accepting $\varphi$. To see this, consider Figure 2:

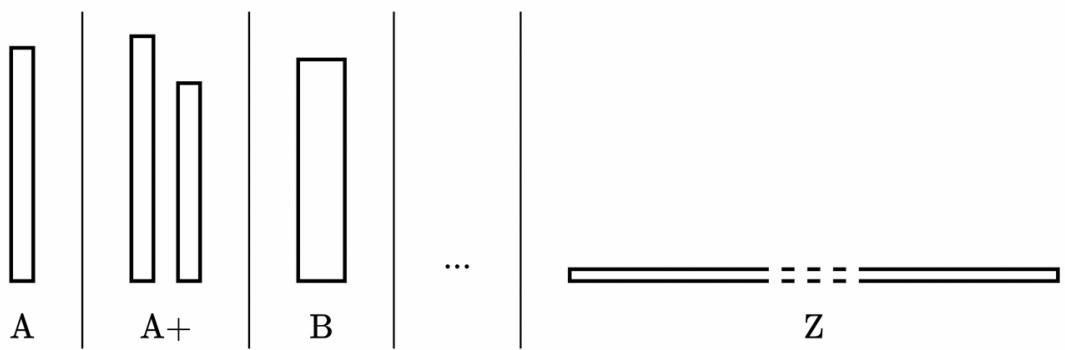

\section{Figure 2}

By accepting $\Pi$ and $\omega$ one accepts that there is some population with some number of lives (however big) with only slightly worse lives that is better than a population with many people with good lives and that the better than relation is transitive, we are forced to conclude that $\mathrm{A}$ is worse than $\mathrm{Z}$. The difference in this argument is that 
one does not move directly from $\mathrm{A}$ to $\mathrm{B}$, from $\mathrm{B}$ to $\mathrm{C}$, etc., but from $\mathrm{A}$ to $\mathrm{A}+$ to $\mathrm{B}$, from $\mathrm{B}$ to $\mathrm{B}+$ to $\mathrm{C}$, etc. Yet, the result is the same: $\mathrm{A}$ is considered worse than $\mathrm{Z}$, which is the Repugnant Conclusion.

There are several proposals of how to avoid the problems that Parfit identifies in the large body of literature that comments on Parfit's arguments (for an overview, see: Arrhenius, Ryberg \& Tännsjö 2010). Some have bitten the bullet and reject that the Repugnant Conclusion is at all repugnant (Tännsjö 2002). A supporting argument for that view might be that we cannot trust our intuitions in cases that involve large numbers (Broome 2004, but see also Pummer 2013 and Temkin 2012). Others have argued that there is some critical level at which an individual's wellbeing no longer contributes positively to the overall value of a population (Blackorby, Bossert \& Donaldson 1995, 1997; Qizilbash 2007). By introducing such a critical level, one can reject the general validity of $\varphi$ and $\omega$ and say that these premises are only valid for comparisons of certain populations, when individuals have wellbeing above the critical level. Yet others have suggested that the positive contribution of an individual's wellbeing to the overall value of a population is diminishing the more people exist in a population ( $\mathrm{Ng} 1989$ ). On this view, $\varphi$ and $\Pi$ are rejected on the basis that both these premises falsely assume that the value of a person's wellbeing is independent of how many people exist, and at some point in the continuum the addition of more people will imply a smaller overall value of the population since it entails a smaller value for the wellbeing of a large number of people. Finally, some have suggested that the take-home lesson rather is that there can be no satisfactory population axiology (Arrhenius MS), i.e. no "betterness ordering of states of affairs, where the states of affairs include ones in which different numbers of persons are ever born" (Greaves 2017: 1).

Neither of these proposals is particularly appealing. Without going into too much detail, it can be noted that they all rely on accepting some deeply counterintuitive view. Accepting that the Repugnant Conclusion is in fact not repugnant at all but just the result of sound inference of the application of appealing normative views goes against almost unanimous considered judgment. Introducing a critical level at which wellbeing no longer contributes positively to the value of a population implies a radical and implausible difference between wellbeing levels which are almost identical (cf. Arrhenius 2004). Depending on how one discounts the value of an individual's wellbeing, this view has very problematic implications. For example, Yew-Kwang Ng's proposal implies that for any number of tormented lives, there are situations in which it is better to add them rather than some people with very good lives (Arrhenius 2000). Rejecting the possibility of a satisfactory population axiology, finally, seems rash since we clearly seem able to order some states of affairs in which different number of people are ever born. 
In the following sections, I will outline what I take to be a plausible way of avoiding the Repugnant Conclusion based on Parfit's latest publications on this issue. The second section addresses the possible objection that this proposal fails to deal with even more problematic issues in population ethics, in particular the socalled impossibility theorems. Finally, I discuss the practical implications of the Parfitian view and identify some ways of developing it further.

\section{I}

Building on two of Parfit's most recent papers, one can develop a general approach to how to avoid the Repugnant Conclusion. This approach has two fundamental elements. First, Parfit's argument that it is a mistake to accept what I will call the Determinate Trichotomy Thesis when thinking about the goodness of different outcomes (Parfit 2016). Second, Parfit's proposed substantive principle of what can make an outcome better than another: the Wide Dual Person-Affecting Principle (Parfit 2017).

Consider, first:

The Determinate Trichotomy Thesis: If $x$ and $y$ are comparable with respect to $p$, it is determinately true that $x$ is more $p$ than $y$, that $x$ is less $p$ than $y$, or that $x$ and $y$ are equally as $p$.

The veracity of this thesis is often taken for granted. It is also often tacitly assumed when the premises used in the Continuum Argument and in the Improved Mere Addition Paradox are considered. When comparing Population A with Population $\mathrm{B}$, it is often thought that if one rejects that $\mathrm{B}$ is determinately better than $\mathrm{A}$, then one must be committed to thinking that in so far as A and $\mathrm{B}$ at all can be compared with respect to how good they are, B is determinately worse than A, or A and B are determinately equally good. This would follow if the Determinate Trichotomy Thesis were true.

However, the Determinate Trichotomy Thesis has been increasingly questioned lately. Some have argued that the trichotomy better than, worse than and equally as good as does not exhaust the set of possible positive value relations (Carlson 2010; Chang 2002, 2016). On this view, it might be true that $x$ and $y$ are comparable with respect to $p$, but not true that either is more $p$ than the other or that they are equally as $p$. Instead, proponents of this view hold that some non-conventional comparative relation such as parity might obtain between $x$ and $y$ with respect to $p$. Others question the Determinate Trichotomy Thesis because they believe that it is some- 
times indeterminate whether $x$ is more $p$ than $y$, less $p$ than $y$ or equally as $p$ as $y$ (Broome 1997, 2004; Contantinescu 2014; Dougherty 2014; Elson 2017). Indeterminacy of this kind is possible if $p$ is vague.

Parfit rejects the Determinate Trichotomy Thesis but presents a different kind of argument for this. He argues that it is sometimes the case that $x$ and $y$ are imprecisely equally as $p$ (which is to be distinguished from being equally as $p$ ). What Parfit means with this is somewhat unclear, but it is clear that he accepts that there are only three kinds of positive relations and he does not think that imprecision can be explained with vagueness:

[T] here are only imprecise truths about the relative goodness of many different acts or outcomes, such as ones that would greatly benefit a few people, or give lesser benefits to many others. Such imprecision is not the result of vagueness in our concepts, or our lack of knowledge, but is part of what we would know if we knew the full facts. When two things are qualitatively very different, these differences would often make it impossible either that one of these things is better than the other by some precise amount, or that both things are precisely equally good (Parfit 2016: 113).

What is important is that contrary to the relation equally as $p$, imprecisely equally as $p$ is not transitive. If $x$ is equally as $p$ as $y$, and $y$ is equally is $p$ as $z$, then $x$ and $z$ are equally as $p$. If $x$ is imprecisely equally as $p$ as $y$, and $y$ is imprecisely equally is $p$ as $z$, then $x$ is not necessarily imprecisely equally as $p$ as $z$.

The introduction of non-transitivity is something Parfit's proposal shares with the non-conventional comparative relations and the indeterminacy proposals, and as we will see this is also a key to avoiding the Repugnant Conclusion. Parity is by definition non-transitive (Chang 2002). Those who believe that it is sometimes indeterminate whether $x$ is more $p$ than $y$ rejects transitivity in a more subtle way. They do not defend non-transitive comparative relations, but they believe that the following is possible: If it is indeterminate whether $x$ is equally as $p$ as $y$, and indeterminate whether $y$ is equally is $p$ as $z$, then it is not necessarily the case that it is indeterminate whether $x$ is equally as $p$ as $z$.

Rather than espousing Parfit's particular view and accepting the possibility of imprecise equality, I believe that it is more fruitful to focus on the negative view, the rejection of the Determinate Trichotomy Thesis. There are competing explanations for why the Determinate Trichotomy Thesis must be rejected (e.g. the possibility of parity, indeterminacy, impreciseness), but how one explains this has little impact on what a plausible population axiology looks like. The take-home lesson is that rejecting the Determinate Trichotomy Thesis might help one solve problems in 
population ethics. I will in what follows say that someone who rejects the Determinate Trichotomy Thesis accepts nondeterminacy, and accepts that alternatives sometimes are nondeterminate in their ranking (cf. Herlitz 2019). Everyone who rejects the Determinate Trichotomy Thesis should accept nondeterminacy, regardless of why they reject the Determinate Trichotomy Thesis.

Rejecting the Determinate Trichotomy Thesis has significant implications. Consider what we, following Joseph Raz's and Muzaffar Qizilbash's discussions of incommensurability and parity and the widespread use of the so-called small improvement argument in the literature on parity, might call a Mark of Nondeterminacy (cf. Chang 2002; Qizilbash 2007; Raz 1986):

A Mark of Nondeterminacy: If $x$ and $y$ are nondeterminate in their ranking with respect to $p$, then an improvement (worsening) in $x$ will not necessarily make the resulting $x$ more (less) $p$ than $y$.

A characteristic feature of nondeterminacy is that if it is true that $x$ is not determinately worse than $y$, and also true that $y$ is not determinately worse than $y$, then an improvement in $x$ does not necessarily make the resulting $x(x+)$ better than $y$. This distinguishes nondeterminacy from equality. If $x$ and $y$ are determinately equally as good, then an improvement in $x$ will necessarily make the resulting $x(x+)$ better than $y$. This is so because equally as good as is a transitive relation so if $x+$ was equally as good as $y$ it would be equally as good as $x$, but it is by definition better than $x$.

Thus, by rejecting the Determinate Trichotomy Thesis Parfit rejects the idea that transitive relations that apply determinately can always rank alternatives with respect to how good they are. This entails accepting the implications of the Mark of Nondeterminacy, that sometimes when neither of two alternatives is determinately worse than the other and one introduces an improvement in one of them, this does not necessarily result in that the resulting, improved alternative is better than the other.

Consider, second:

The Wide Dual Person-Affecting Principle: One of two outcomes would be in one way better if this outcome would together benefit people more, and in another way better if this outcome would benefit each person more (Parfit 2017: 154).

Some explanation of this principle is required. The context in which Parfit presents this view is a discussion of the so-called Non-Identity Problem. Like the Repugnant 
Conclusion, this is a notorious problem in population ethics that was identified by Parfit in his earlier work and which has generated a large body of literature (Parfit 1984; Roberts 2015). A fundamental question in the debate on the Non-Identity Problem is whether something can be good without being good for someone, and in particular whether some benefit can be good if the person benefitting would not exist had it not been for the benefit. In his last paper on this issue, Parfit espouses a view presented by Jeff McMahan:

If someone is caused to exist and to have a life that is worth living, that is good for this person, giving him or her an existential benefit. There are similar existential harms (McMahan 2013: 6-7).

This is what Parfit has in mind when he claims that his principle is a wide personaffecting principle (as opposed to a narrow person-affecting principle which rejects the possibility of existential benefits). It is a principle according to which nothing can be good (bad) unless it is good (bad) for someone, but this is understood so that it can be good (bad) for a person to be caused to exist.

Next, Parfit distinguishes between two senses in which one of two outcomes might benefit people more. An outcome would:

(1): benefit people more in the collective sense if this outcome would together benefit people more.

(2): benefit people more in the individual sense if this outcome would benefit each person more (Parfit 2017: 152).

Parfit believes that an outcome or population can be better than another in both of these senses. First, he accepts that if one of two outcomes contains a greater total sum of wellbeing, it is in one way better; it benefits people more in the collective sense. Second, he accepts that if one of two outcomes benefits each person more and thereby is better for every individual in the outcome, it is in one way better; it benefits people more in the individual sense. Thus, the Wide Dual Person-Affecting Principle states that one of two outcomes would be in one way better if the total amount of wellbeing is greater, and in another way better if this outcome would benefit each person more.

It is clear that some outcomes are better than others in the collective sense but not in the individual sense. This is true, for instance, of the populations in the continuum of populations that are compared in the Continuum Argument. Population 
$\mathrm{B}$ is better than Population A in the collective sense, but not in the individual sense.

Importantly, if one - like Parfit - rejects the Determinate Trichotomy Thesis one is not committed to the idea that we can always determine which of two outcomes is at least as good as the other overall when one outcome is better than another in one respect but not in the other respect. Instead, one can accept that two outcomes might be nondeterminate in their ranking with respect to the collective and the individual senses of benefit. In other words, the Wide Dual Person-Affecting Principle allows for nondeterminacy and fails to always fully determine an outcome that is at least as good as all alternative outcomes.

In combination, rejecting the Determinate Trichotomy Thesis and accepting the Wide Dual Person-Affecting principle tell us that: an outcome is (i) in one way better than another if it would together benefit people more and (ii) in another way better if it would benefit each person more, but (iii) we should not assume that we can always fully determine that any of the following relations obtain between two outcomes: better than, worse than, equally as good as.

We now have the tools to dismiss both the Continuum Argument and the Improved Mere Addition Paradox, and what transpires is a thus a view that seems to avoid the Repugnant Conclusion. Both the Continuum Argument and the Improved Mere Addition Paradox rely on the tacit assumption that one can rank the populations under consideration with transitive relations, so rejecting this is in itself sufficient to dismiss both these arguments. However, in order to explain why the premises that these arguments rely on are appealing and how this can be accounted for, one needs a substantive view of what makes an outcome better than another. The Wide Dual Person-Affecting Principle is such a view.

Consider first the Continuum Argument. This argument can be dismissed on the grounds that it mistakenly assumes that if an outcome is significantly better than another in one way, it can always be determined that it is all things considered better. For some populations next to each other in the continuum, we might say that it will be false that either is at least as good as the other. Instead, it will be the case that they are nondeterminate in their ranking. These populations next to each other in the continuum will be nondeterminate in their ranking because one will be better than the other in the collective sense, but not in the individual sense, and in some situations it is impossible to fully determine that either of two outcomes is at least as good as the other with respect to these considerations. Since nondeterminacy is a non-transitive relation, this breaks the series of inferences that leads one from holding that $\mathrm{B}$ is better than $\mathrm{A}$ to $\mathrm{Z}$ is better than $\mathrm{A}$. It allows one to, for instance, hold the view that $\mathrm{A}$ is worse than $\mathrm{B}$, that $\mathrm{B}$ and $\mathrm{C}$ are nondeterminate in their ranking, but that $\mathrm{A}$ is better than $\mathrm{C}$.

Similarly, the Improved Mere Addition Paradox can be dismissed with reference 
to how one of its premises $(\omega)$ mistakenly assumes that if an outcome is significantly better than another in one way, it can always be determined that it is all things considered better. For some populations with features that are similar to Populations $\mathrm{A}+$ and $\mathrm{B}$, where the populations are equally big, the first population contains inequality and the second population is perfectly equal, contains more wellbeing, but the best off are worse off, it will be false that either is at least as good as the other. Instead, it will be the case that they are nondeterminate in their ranking. These populations next to each other in the continuum will be nondeterminate in their ranking because one will be better than the other in the collective sense, but not in the individual sense, and in some situations it is impossible to fully determine that either of two outcomes is at least as good as the other with respect to these considerations. Again, since nondeterminacy is a nontransitive relation, this breaks the series of inferences that leads one from holding that $\mathrm{B}$ is better than $\mathrm{A}$ to $\mathrm{Z}$ is better than $\mathrm{A}$.

The issue of where on the continuum the nondeterminacy occurs relates to how one specifies the Wide Dual Person-Affecting Principle. Parfit provides no specification of this principle, but it can be recognized that there is a wide range of possible specifications compatible with Parfit's general proposal. There are at least three dimensions in which the Wide Dual Person-Affecting Principle can be specified: (i) what is the relative value of collective benefits compared to individual benefits; (ii) how wide is the scope of nondeterminacy, i.e. how many outcomes will the principle fail to determinately rank trichotomously; (iii) where on the continuum does the nondeterminacy occur, i.e. does it arise when collective benefits are high (low) and/or when individual benefits are high/low?

A general conclusion can now be drawn by a Parfitian approach to how one can avoid the Repugnant Conclusion: A population axiology can avoid the Repugnant Conclusion while it accounts for the underlying intuitions that otherwise lead to the Repugnant Conclusion if these intuitions are reflected in a principle that allows for nondeterminacy. This is compatible with various views of why nondeterminacy arises. This might be due to non-conventional comparative relations such as parity, vagueness, or what Parfit referred to as impreciseness. It is also compatible with a variety of substantive principle, including various specifications of the Wide Dual Person-Affecting Principle.

\section{II}

In a paper that comments on Parfit's proposal of how to avoid the Continuum Argument and the Improved Mere Addition Paradox by rejecting the Determinate Trichotomy Thesis, Gustaf Arrhenius raises another concern: even if one rejects the 
Determinate Trichotomy Thesis, it is not clear how one can deal with the impossibility theorems that show that there can be no satisfactory theory of population ethics (Arrhenius 2016). In this section, I outline a response to Arrhenius based on the Parfitian approach to how to avoid the Repugnant Conclusion.

Arrhenius has presented six different theorems in which he shows that no population axiology can meet a set of highly plausible adequacy conditions (Arrhenius MS). Of these, Arrhenius believes that the sixth theorem is the strongest (Arrhenius 2016). I will here focus on the strongest theorem:

Arrhenius's Sixth Impossibility Theorem: There is no population axiology which satisfies the Egalitarian Dominance, the General Non-Extreme Priority, the NonElitism, the Weak Non-Sadism, and the Weak Quality Addition Condition (Arrhenius 2009).

Arrhenius argues that there is no population axiology that satisfies the following adequacy conditions (here presented in the informal way):

The Egalitarian Dominance Condition: If population A is a perfectly equal population of the same size as population $\mathrm{B}$, and every person in $\mathrm{A}$ has higher welfare than every person in B, then $\mathrm{A}$ is better than $\mathrm{B}$, other things being equal.

The General Non-Extreme Priority Condition: There is a number $n$ of lives such that for any population $\mathrm{X}$, and any welfare level $\mathrm{A}$, a population consisting of the $\mathrm{X}$-lives, $n$ lives with very high welfare, and one life with welfare $\mathrm{A}$, is at least as good as a population consisting of the X-lives, $n$ lives with very low positive welfare, and one life with welfare slightly above A, other things being equal.

The Non-Elitism Condition: For any triplet of welfare levels, A, B, and C, A slightly higher than B, and B higher than C, and for any one-life population A with welfare $A$, there is a population $C$ with welfare $C$, and a population $B$ of the same size as A $\cup$ C and with welfare B, such that for any population $\mathrm{X}$ consisting of lives with welfare ranging from $\mathrm{C}$ to $\mathrm{A}, \mathrm{BUX}$ is at least as good as AUCUX, other things being equal.

The Weak Non-Sadism Condition: There is a negative welfare level and a number of lives at this level such that an addition of any number of people with positive welfare is at least as good as an addition of the lives with negative welfare, other things being equal. 
The Weak Quality Addition Condition: For any population X, there is a perfectly equal population with very high positive welfare, and a very negative welfare level, and a number of lives at this level, such that the addition of the high welfare population to $\mathrm{X}$ is at least as good as the addition of any population consisting of the lives with negative welfare and any number of lives with very low positive welfare to X, other things being equal (Arrhenius 2009: 28-30).

Arrhenius proves that no population axiology can meet all five adequacy conditions. This holds also for the Parfitian view, and so one wonders which of the adequacy conditions to reject.

In light of the Wide Dual Person-Affecting Principle, three of the conditions seem indisputably valid. The Egalitarian Dominance condition states that if two populations have the same size, there is perfect equality in both populations, and the wellbeing of the people in one population is greater than the wellbeing of the people in the other, then the population with the better off people is better. The Wide Dual Person-Affecting Principle is in complete agreement with this. Since both the total amount of wellbeing is higher and everyone is better off in one of the populations, this population is better in both of the respects that the Wide Dual Person-Affecting Principle recognizes. Also, the Weak Non-Sadism Condition is clearly met by the Wide Dual Person-Affecting Principle, since this principle implies that adding people with negative wellbeing is bad and that adding people with positive wellbeing is good. Finally, the Weak Quality Addition Condition is met by the Parfitian view since it is a weaker formulation of avoidance of the Repugnant Conclusion (cf. Arrhenius 2016; Fehige 1998). Thus, even if the Wide Dual Person-Affecting Principle does not in itself necessarily meet this condition, one must be committed to a specification of this principle that meets the condition in so far as one wants to avoid the Repugnant Conclusion.

Those accepting the Parfitian view might, however, reject either the General Non-Extreme Priority Condition or the Non-Elitism Condition, or both. To see this, consider how both of these conditions restrain what a population axiology must say about comparisons of different distributions where it is the case that some are better off in one distribution and some are better off in a different distribution. The Parfitian view of the implications of the Wide Dual Person-Affecting Principle clearly is that in some cases in which some are better off in one outcome and some are better off in a different outcome the two outcomes are nondeterminate in their ranking. This is how one dismisses the Improved Mere Addition Paradox. Those accepting the Parfitian view might, thus, argue that either one or both of these conditions mistakenly requires of a population axiology that it implies that if an outcome is significantly better than another in some but not all ways, it can always 
be determined that it is all things considered better. If one is inclined to believe that nondeterminacy arises when the worst off in a population is worse off in one of the outcomes, one might reject the General Non-Extreme Priority Condition. If one is inclined to believe that nondeterminacy arises when the best off in a population is worse off in one of the outcomes, one might reject the Non-Elitism Condition. If one believes that nondeterminacy is plausible in both kinds of situation, one might reject both conditions. The Wide Dual Person-Affecting Principle is compatible with all of these positions. Which one to go for depends on how one thinks the principle should be specified.

In light of this, one might suggest that Arrhenius makes a mistake when he formulates his adequacy condition by ruling out nondeterminacy. To allow for nondeterminacy, either or both of the following can instead be used as adequacy conditions:

The General Non-Extreme Priority Condition*: There is a number $n$ of lives such that for any population $\mathrm{X}$, and any welfare level $\mathrm{A}$, a population consisting of the $\mathrm{X}$-lives, $n$ lives with very high welfare, and one life with welfare $\mathrm{A}$, is not determinately worse than a population consisting of the $\mathrm{X}$-lives, $n$ lives with very low positive welfare, and one life with welfare slightly above $\mathrm{A}$, other things being equal.

The Non-Elitism Condition*: For any triplet of welfare levels, A, B, and C, A slightly higher than B, and B higher than C, and for any one-life population A with welfare $A$, there is a population $C$ with welfare $C$, and a population $B$ of the same size as $A \cup C$ and with welfare $B$, such that for any population $X$ consisting of lives with welfare ranging from $\mathrm{C}$ to $\mathrm{A}, \mathrm{BUX}$ is not determinately worse than AUCUX, other things being equal.

These definitions capture the general intuitions behind the adequacy conditions, aversion to extreme priority to the worst off and to elitism respectively, while they allow for nondeterminacy. However, they do not (together with the other adequacy conditions) rule out the possibility of a population axiology. To see this, it suffices to note that the Wide Dual Person-Affecting Principle can be understood so that it meets all five adequacy conditions if one replaces either the non-elitism condition or the non-extreme priority condition as suggested above. It can be noted that this revision is also in line with some other work on population ethics, in particular the approaches that want to allow for indeterminacy due to vagueness (Broome 2004; Qizilbash 2007, 2018; Thomas 2015).

It could of course be objected that these revisions of the adequacy conditions 
imply that they lose significant intuitive support. The aversion to extreme priority to the worst off and to elitism indicates that there are some outcomes that are better than the outcomes in which the worst off/best off fares best. The revised adequacy conditions fail to reflect this.

In response to this objection, and in the spirit of Parfit's latest work, it is worth pointing out that the question we must ask is not whether it is in itself plausible to revise these conditions. Instead, the relevant question is whether it is more implausible to make these revisions than to accept that there can be no satisfactory population axiology. Making these revisions seems to me to be the least implausible of these options.

Furthermore, there might be further reasons to reject the Determinate Trichotomy Thesis and accept revisions of Arrhenius adequacy conditions of this kind. For the Determinate Trichotomy Thesis to be true it is not enough that the normative principles that apply are able to in principle provide determinate trichotomous orderings. It must also be possible to order the elements that these principles order with respect to their pertinent characteristics. In population ethics, two characteristics are generally considered pertinent when one considers different populations: size and wellbeing. Of course, size is measured by natural numbers and natural numbers by their nature allow for determinate, trichotomous orderings. It is determinately true for all pairs of natural numbers that one is greater than the other or that they are of equal size. It is, however, far from obvious that one can say the same about wellbeing. Instead, it might be the case that two lives are nondeterminate in their ranking with respect to wellbeing. This might, for instance, be a plausible conclusion when one compares one life that is short but filled with achievements and happiness with a life that is long but filled with health problems. If lives with different properties cannot be determinately, trichotomously ordered with respect to wellbeing, this challenges the assumption that populations can be determinately, trichotomously ordered with respect to how good they are. If one rejects the Determinate Trichotomy Thesis on the grounds that wellbeing levels cannot be determinately, trichotomously ranked, this gives one further reason to allow for nondeterminacy and revise Arrhenius's adequacy conditions.

\section{III}

The following general hypothesis can now be introduced in light of Parfit's approach to how to avoid the Repugnant Conclusion and the discussion of Arrhenius's impossibility theorem: 
Nondeterminate Population Ethics: An approach to population axiology can only be satisfactory if it allows for nondeterminacy.

It might be argued that one concedes too much by giving up of the Determinate Trichotomy Thesis since this implies that one loses the ability to make practical judgments with the normative principles one accepts. If one's principles only manage to establish that some alternatives are nondeterminate in their ranking, it is hard to see what this theory prescribes. There is a sense in which a theory that allows for nondeterminacy is unsatisfactory.

This worry is misguided. Depending on how one explains nondeterminacy, one can use principles that allow for nondeterminacy to weed out impermissible alternatives (cf. Herlitz 2019). Conceptualizing nondeterminacy in terms of incompleteness allows one to use Amartya Sen's conception of maximization and say that all alternatives that are not maximal (i.e. all alternatives that are determinately worse than some alternative) are impermissible (Sen 1997, 2017). Thinking of nondeterminacy in terms of vagueness enables one to say that all alternatives that are worse than some alternative on some admissible precisification are impermissible (Andersson 2017; Broom 2009; Fine 1975). Using the fitting attitudes approach to value, one might stipulate that all alternatives that are impermissible to prefer or equiprefer to some alternative are impermissible (cf. Rabinowicz 2008).

I will not suggest that any of these approaches is preferable to the others, but it is important to note that there are several ways in which principles that allow for nondeterminacy can be used to create partial orderings. This reveals a significant advantage that this approach has to skepticism about the possibility of a satisfactory population axiology. The skeptic cannot give any explanation of why a population with many people who lead mediocre lives is worse than a population with the exact same people in it with better lives. A principle that allows for nondeterminacy has no problems determining that it is better if everyone lead better life.

Nevertheless, a problem will remain for proponents of principles that allow for nondeterminacy: in some situations, two or more alternatives in the feasible choice set will remain after one has discarded impermissible alternatives. How to choose between alternatives that are nondeterminate in their ranking is no small problem. Should we consider them equally permissible on the ground that they all passed the impermissibility test? Or should we introduce some further condition? Following Sen, one might come to the conclusion that one can rationally choose any option that is not determinately worse than any alternative (Sen 1997). Following Ruth Chang, one might instead recognize that when two alternatives are nondeterminate in their ranking there is sometimes what Chang calls a "resolutional remainder" that implies that further reasons are needed to justify a choice (Chang 2002). When two 
alternatives are nondeterminate in their ranking people can reasonably disagree about which is better, and reasons apply to this disagreement. This question must be given more attention by population ethicists who accept nondeterminacy.

Regardless of what one thinks about whether or not further reasons are needed to choose between alternatives that are nondeterminate in their ranking, there might be formal constraints that apply to such choices. To see this, consider a wellknown problem that arises as soon as one rejects the Determinate Trichotomy Thesis: sequences of choice situations in which some alternatives are nondeterminate in their ranking can sometimes be determinately, trichotomously ranked even if alternatives in each step of the sequences cannot be determinately, trichotomously ranked (cf. Elga 2010; McClennen 1990).

Consider an illustration. Assume that $\mathrm{A}$ and $\mathrm{B}$ are nondeterminate in their ranking, that $\mathrm{A}$ is worse than $\mathrm{A}+$, and that $\mathrm{A}+$ and $\mathrm{B}$ are nondeterminate in their ranking. Assume separability so that the values of $\mathrm{A}, \mathrm{A}+$ and $\mathrm{B}$ in the two choice situations are independent of other choices:

\begin{tabular}{|l|l|l|}
\hline \multicolumn{1}{|c|}{ Choice 1 } & Choice 2 \\
\hline Sequence $X$ & A & B \\
\hline Sequence $Y$ & B & A+ \\
\hline
\end{tabular}

\section{Table 1}

We can easily see that Sequence $\mathrm{X}$ is determinately worse than Sequence $\mathrm{Y}$ since A+ is determinately better than $\mathrm{A}$, and the only difference in value between the sequences is that one includes $\mathrm{A}$ and the other $\mathrm{A}+$ (remember that we have assumed separability).

In light of this phenomenon, it can be suggested that whatever approach one takes to alternatives that are nondeterminate in their ranking, this approach should rule out the possibility of making sequences of choices that are determinately worse than some other sequence. However one formulates such a criterion, it constrains how to make decisions in face of nondeterminacy. For instance, it rules out the view that one can permissibly flip a coin between alternatives that are nondeterminate in their ranking, but it does not rule out the view that one should always favor A-type values over B-type values when some alternatives are nondeterminate in their ranking. Population ethicists that accept nondeterminacy should give more attention to what kinds of formal criteria one can pose of decision methods when the normative grounds for the decisions allow for nondeterminacy. 


\section{IV}

Parfit's last work on population ethics teaches us that we can avoid the Repugnant Conclusion if we reject the Determinate Trichotomy Thesis and accept nondeterminacy. This also provides us with the tools necessary to pose relevant adequacy conditions on what a satisfactory population axiology is that allow for the possibility of a population axiology. A plausible population axiology is a population axiology that only partially orders outcomes. A particular question that arises when we accept this is: to what extent can one introduce formal constraints on what practical judgments are permissible with respect to a population axiology that allows for nondeterminacy? Nondeterminacy does not pose a general challenge to the possibility of forming practical judgments based on one's population axiology, but it means that we should think of practical judgments in population ethics in unconventional ways.

\section{References}

Andersson, Henrik. 2017. How it all relates: Exploring the space of value comparisons. Ph.D. dissertation, Lund University.

Arrhenius, Gustaf. 2000. “An Impossibility Theorem for Welfarist Axiology,” Economics and Philosophy, 16: 247-266.

Arrhenius, Gustaf. 2004. "Superiority in Value,” Philosophical Studies, 123: 97-114. Arrhenius, Gustaf. 2009. “One More Axiological Impossibility Theorem,” in LarsGöran Johansson, Jan Österberg and Rysiek Sliwinski (eds.), Logic, Ethics, and All That Jazz: Essays in Honour of Jordan Howard Sobel. Uppsala: Uppsala University: 23-37.

Arrhenius, Gustaf. 2016. "Population Ethics and Different-Number-Based Imprecision,” Theoria, 82(2): 166-181.

Arrhenius, Gustaf. MS. Population Ethics: The Challenge of Future Generations.

Arrhenius, Gustaf, Jesper Ryberg and Torbjörn Tännsjö. 2010. “The Repugnant Conclusion,” in E. N. Zalta (ed.) The Stanford Encyclopedia of Philosophy.

Blackorby, Charles, Walter Bossert and David Donaldson. 1995. "Intertemporal Population Ethics: Critical-Level Utilitarian Principles,” Econometrica, 63(6): 1303-1320.

Blackorby, Charles, Walter Bossert and David Donaldson. 1997. "Critical-Level Utilitarianism and the Population-Ethics Dilemma,” Economics and Philosophy, 13(2): 197-230. 
Broome, John. 1997. “Is Incommensurability Vagueness?” in Ruth Chang (ed.), Incommensurability, Incomparability, and Practical Reason. Cambridge, MA: Harvard University Press.

Broome, John. 2004. Weighing Lives. Oxford: Oxford University Press. Broome, John. 2009. "Reply to Rabinowicz,” Philosophical Issues 19: 41--417. Carlson, Erik. 2010. "Parity Demystified.” Theoria. 76, 119-128.

Chang, Ruth. 2002. “The Possibility of Parity,” Ethics, 112(4): 659-688.

Chang, Ruth. 2016. "Parity, Imprecise Comparability and the Repugnant Conclusion,” Theoria, 82(2): 182-214.

Contantinescu, Cristian. 2014. "Moral Vagueness: A Dilemma for NonNaturalism,” in Russ Shafer-Landau (ed.), Oxford Studies in Metaethics, Volume 9. Oxford: Oxford University Press: 152-185.

Dougherty, Tom. 2014. "Vague Value," Philosophy and Phenomenological Research, 89(2): 352-372.

Elga, Adam. 2010. “Subjective Probabilities Should be Sharp,” Philosopher's Imprint 10: 1-11.

Elson, Luke. 2017. "Incommensurability as Vagueness: A Burden-Shifting Argument.” Theoria. 83: 341-363.

Fehige, Christoph. 1998. “A Pareto Principle for Possible People,” in Christop Fehige and Ulla Wessels (eds.), Preferences, Perspektiven der analytischen Philosophie; Perspectives in analytical philosophy. Berlin: W. de Gruyter: 508-543. Fine, Kit. 1975. "Vagueness, Truth and Logic," Synthese, 30: 265-300.

Greaves, Hilary. 2017. “Population Axiology,” Philosophy Compass, 12(11): e12442. Herlitz, Anders. 2019. "Nondeterminacy, two-step models and justified choice." Ethics, 129(2): 284-308.

McClennen, Edward F. 1990. Rationality and Dynamic Choice: Foundational Explorations. Cambridge: Cambridge University Press.

McMahan, Jeff. 2013. “Causing People to Exist and Saving People's Lives,” Journal of Ethics, 17(1): 5-35.

Ng, Yew-Kwang. 1989. "What Should We Do about Future Generations? The Impossibility of Parfit's Theory X,” Economics and Philosophy, 5(2): 235-253.

Parfit, Derek. 1982. "Future Generations: Further Problems," Philosophy and Public Affairs, 11: 113-172.

Parfit, Derek. 1984. Reasons and Persons. Oxford: Clarendon Press.

Parfit, Derek. 2016. “Can We Avoid the Repugnant Conclusion?” Theoria, 82(2): 110-127. 
Parfit, Derek. 2017. "Future People, the Non-Identity Problem, and PersonAffecting Principles,” Philosophy and Public Affairs, 45(2): 118-157.

Pummer, Theron. 2013. "Intuitions about large number cases," Analysis, 73: 37-46. Qizilbash, Mozaffar. 2007. “The Mere Addition Paradox, Parity and Vagueness,” Philosophy and Phenomenological Research, 75(1): 129-151.

Qizilbash, Mozaffar. 2018. “On Parity and the Intuition of Neutrality,” Economics and Philosophy, 34: 87-108.

Rabinowicz, Wlodek. 2008. "Value Relations,” Theoria, 74: 18-49.

Rachels, Stuart. 1998. “Counterexamples to the Transitivity of Better Than," Australasian Journal of Philosophy, 76: 71-83.

Raz, Joseph. 1986. The Morality of Freedom. Oxford: Oxford University Press.

Roberts, M. A. 2015. “The Nonidentity Problem,” in E. N. Zalta (ed.) The Stanford Encyclopedia of Philosophy.

Sen, Amartya. 1997. "Maximization and the Act of Choice," Econometrica, 65: 745779.

Sen, Amartya. 2017. "Reason and Justice: The Optimal and the Maximal," Philosophy, 92: 5-19.

Temkin, Larry. 1987. "Intransitivity and the Mere Addition Paradox," Philosophy and Public Affairs, 16: 138-187.

Temkin, Larry. 2012. Rethinking the Good: Moral Ideals and the Nature of Practical Reasoning. Oxford: Oxford University Press.

Thomas, Teruji. 2017. "Some Possibilities in Population Axiology,” to appear in Mind. DOI:10.1093/mind/fzx047

Tännsjö, Torbjörn. 2002. "Why We Ought to Accept the Repugnant Conclusion," Utilitas, 14(3): 339-359. 